\title{
Decent Work und die Universität
}

\author{
Sebastian SCHIEF ${ }^{1}$ \\ Universität Fribourg
}

Mit der Entwicklung des Konzeptes der Decent Work hat die International Labour Organisation (ILO) im Jahre 1999 versucht, Standards für eine menschenwürdige Arbeit zu formulieren, um weltweit verbesserte Arbeitsbedingungen zu erreichen. Das Konzept ist über verschiedene wichtige Wegmarken entwickelt worden (vgl. Ferraro et al. 2016). Dies begann selbstverständlich mit der Gründung der ILO im Jahr 1919, der Deklaration der Ziele und Prinzipien der ILO im Jahr 1944 (ILO 1944) und deren Konstitution (ILO 1946). Das Konzept verdeutlicht, dass für die ILO Erwerbsarbeit keine Ware ist und soziale Gerechtigkeit, Freiheit, Würde, ökonomische Sicherheit und Chancengleichheit im Zentrum stehen. Menschenwürdige Arbeit stehe demnach in engem Zusammenhang zu den fundamentalen Menschenrechten. In der Erklärung der Menschenrechte (1948) findet sich dann auch ein Passus, der die freie Wahl der Arbeitsstelle, gerechte und angemessene Bedingungen der Arbeit und den Schutz vor Arbeitslosigkeit hervorhebt (United Nations 2015). Zu diesen fairen Arbeitsbedingungen gehören eine angemessene Bezahlung, das Recht, Gewerkschaften zu gründen und beizutreten, aber auch soziale Sicherung.

Im Jahr 1999 hat die ILO das Konzept der Decent Work lanciert und als primäres Ziel der ILO formuliert, „to promote opportunities for women and men to obtain decent and productive work, in conditions of freedom, equity, security and human dignity" (ILO 1999). Inzwischen wurde das Konzept auch als eines der 17 Ziele der Agenda Nachhaltige Entwicklung der Vereinten Nationen 2030 deklariert. Diese Ziele werden auch vom Kanton Fribourg aktiv unterstützt.

Menschenwürdige Arbeit wird folgendermassen definiert:

Decent work is defined by the ILO and endorsed by the international community as productive work for women and men in conditions of freedom, equity, security and human dignity. Decent work involves opportunities for work that: is productive and delivers a fair income; provides security in the workplace and social protection for workers and their families; offers prospects for personal development and encourages social integration; gives people the freedom to express their concerns, to organize and

1 PD Dr. Sebastian Schief ist Soziologe und arbeitet im Bereich Soziologie, Sozialpolitik, Sozialarbeit der Universität Fribourg/Schweiz (sebastian.schief@unifr.ch). 
to participate in decisions that affect their lives; and guarantees equal opportunities and equal treatment for all. (ILO 2008b: iv)

Das Konzept der menschenwürdigen Arbeit basiert auf vier normativen Bedingungen: der Schaffung von Arbeit, den Rechten bei der Arbeit, sozialer Sicherung und Sozialpartnerschaft (ILO 2008). Mit dem Konzept der Decent Work wurde also ein normativer Rahmen geschaffen, hinter den auf lange Sicht keine Arbeitsstelle zurückfallen sollte.

Schmucker (2020) weist in dieser Ausgabe von sozialpolitik.ch darauf hin, dass die Standards von Decent Work grundlegende Anforderungen und Mindeststandards an Arbeit definieren, die für hochentwickelte Staaten wie Deutschland demnach notwendige aber keineswegs hinreichende Bedingungen sind. Um diesen Gedanken Folge zu leisten, wurde vom Deutschen Gewerkschaftsbund das Konzept Gute Arbeit entwickelt, das in seinen Anforderungen an die Qualität der Arbeit höhere und ausdifferenzierte Standards als jene des Konzeptes Decent Work definiert. Basierend auf einer Befragung von abhängig Beschäftigen formuliert Fuchs, was aus der Perspektive der Beschäftigten gute Arbeit ist:

'Gute Arbeit' bedeutet aus der Sicht von Arbeitnehmer/-innen, ein festes, verlässliches Einkommen zu erhalten, unbefristet beschäftigt zu sein, die fachlichen und kreativen Fähigkeiten in die Arbeit einbringen und entwickeln zu können, Anerkennung zu erhalten und soziale Beziehungen zu entwickeln. Positiv wird Arbeit bewertet, wenn ausreichend Ressourcen vorhanden sind, z.B. Entwicklungs-, Qualifizierungs-und Einflussmöglichkeiten und gutes soziales Klima zu den Vorgesetzten und Kolleg/-innen. Eine weitere wichtige Bedingung ist, dass das Anforderungsniveau nicht zu stark als belastend empfunden wird. (Fuchs 2006: 8)

Wenn man sich diese Definition guter Arbeit zu eigen macht, kann man anhand der oben genannten Kriterien versuchen, verschiedene Organisationen und Unternehmen daraufhin überprüfen, ob sie aus Sicht ihrer Beschäftigten oder Teile der Beschäftigten diesen Anforderungen genügen. Anhand eines fiktiven Beispiels wird nun im Folgenden versucht zu zeigen, wie verschiedene rechtliche Regelungen und Reglemente des Kantons Fribourg und der Universität Fribourg dazu führen, dass bestimmte Teile der abhängig Beschäftigten der Universität nicht unter Bedingungen arbeiten, die den Standards des Konzeptes Gute Arbeit genügen. Im Einzelnen eingehen werde ich auf fünf wichtige Aspekte, die aus meiner Sicht problematisch sind: eine im Schweizer Vergleich sehr lange Probezeit, eine Ketten-Befristung von Arbeitsverträgen, verschiedene Handhabungen von normalen und maximalen Vertragslängen, ein prekärer Schutz von Beschäftigten im Falle von Krankheit, sowie eine ungeklärte Lage von Beschäftigten in Drittmittel-Projekten im Falle von längerfristigen Krankheiten.

\section{Probezeit und Dauer des Anstellungsverhältnisses}

Stellen wir uns vor, Lena P. hat Ihr Masterstudium in Biologie in Bern erfolgreich abgeschlossen und kann sich vorstellen, in der Wissenschaft zu bleiben und ein Doktorat durchzuführen. Sie bewirbt sich an verschiedenen Universitäten und bekommt schliesslich eine Stelle an der Universität Fribourg angeboten. Lena P. freut sich über das Angebot, sagt schnell zu und bekommt nach einigen Wochen den Arbeitsvertrag zugeschickt. Nach Durchsicht des Arbeitsvertrages 
fällt ihr auf, dass der erste Arbeitsvertrag laut Reglement über die wissenschaftlichen Mitarbeiterinnen und Mitarbeiter (RwMM), Artikel 17, nur für neun bis fünfzehn Monate ausgestellt werden kann. Anschliessend, so das Reglement, „werden die Diplomassistenten und -assistentinnen, deren Besoldung durch das Budget des Kantons finanziert wird, bis zum Ende der von der Fakultät für den entsprechenden Bereich festgelegten üblichen Dauer der Doktorarbeit angestellt“ (Artikel 17, RwMM). Das heisst also für Lena - denn die Mathematisch-Naturwissenschaftliche und Medizinische Fakultät der Universität Fribourg verweist in Ihrem DoktoratsReglement $^{2}$ auf eine übliche Dauer der Doktorarbeit von vier Jahren (Artikel 6, Doktoratsreglement Mathematisch-Naturwissenschaftliche und Medizinische Fakultät der Universität Fribourg) - dass sie einen zweiten Vertrag zwischen maximal 33 und 41 Monaten (je nach Länge des ersten Vertrages) erhielte. Insgesamt beschränkt das Reglement die Anstellungsdauer von DiplomassistentInnen auf 5 Jahre (Artikel 17, RwMM).

Ein weiterer Passus der Unterlagen verweist auf das Reglement der Probezeit für alle Angestellten des Kanton Fribourg. Der Kanton sieht in seinem Gesetz über das Staatspersonal (LPers) eine Probezeit von 12 Monaten vor. ${ }^{3}$ Während der ersten drei Monate gilt eine Frist von 7 Tagen, ab dem vierten Monat handelt es sich um eine Monatsfrist. Zusätzlich kann die Arbeitgeberin im Falle von Zweifeln über die Fähigkeiten des Arbeitnehmers oder der Arbeitnehmerin die Probezeit um ein weiteres Jahr verlängern. Da Lena keine Berufserfahrung aufzuweisen hat, gilt für sie die volle Probezeit von einem Jahr.

Die Dauer der Probezeit im Kanton Fribourg ist im Vergleich zu den meisten der 25 anderen Kantonen ausgesprochen lang. ${ }^{4}$ Das Obligationenrecht (OR) der Schweiz sieht eine Probezeit von einem Monat vor (Artikel 335b, OR). Innerhalb dieser Zeit kann innerhalb von 7 Tagen gekündigt werden. Zwei Kantone haben diese Regelungen übernommen, 15 Kantone kennen eine Frist von 3 Monaten, für 5 Kantone gilt eine Frist von 6 Monaten. Eine Frist von 12 Monaten und mehr gilt ausser in Fribourg nur noch in zwei anderen Kantonen (Uri und Neuchâtel).

Für unseren hypothetischen Fall bedeutet dies alles in allem, dass Lena keinen Vertrag, wie er für Diplomassistenzen maximal vorgesehen ist (5 Jahre), haben wird. Die Doktorandin erhält einen ersten, kurzen Vertrag, dann einen zweiten, längeren Vertrag, beide Verträge zusammen werden im Normalfall vier Jahre nicht überschreiten. Dieser Vertrag kann, muss aber nicht noch einmal verlängert werden. Zusätzlich zu der ohnehin gegebenen Befristung besteht beim ersten Vertrag eine Probezeit, die es der Arbeitgeberin erlaubt, in den ersten drei Monaten in Sieben-Tages-Frist zu kündigen, ab dem vierten Monat dann in Monatsfrist. Vergleichen wir

\footnotetext{
${ }^{2}$ Reglement vom 21. September 2015 (Stand 17. Dezember 2018) über die Erlangung der Doktorwürde der Wissenschaften oder der Philosophie an der Mathematisch-Naturwissenschaftlichen und Medizinischen Fakultät der Universität Freiburg $(\mathrm{PhD})$.

${ }^{3}$ Laut Verwaltung der Universität werden Verträge unter einem Jahr folgendermassen gehandhabt: Verträge bis zu einem Monat Laufzeit haben keine Probezeit; Verträge zwischen einem und drei Monaten haben einen Monat Probezeit, Verträge zwischen 3 und 12 Monaten haben 3 Monate Probezeit (schriftliche Information).

${ }^{4}$ In Artikel 31, Absatz 4, LPers heisst es: „Bei der Anstellung oder während der Probezeit kann ganz oder teilweise auf diese verzichtet werden, und zwar für befristete Verträge, wenn die Mitarbeiterin oder der Mitarbeiter die betreffende Funktion schon vorher ausgeübt hat oder wenn die Leistungen, das Verhalten und die Fähigkeiten mit denen erfahrener Mitarbeiterinnen und Mitarbeiter vergleichbar sind. Die Artikel 34 und 35 bleiben im Übrigen vorbehalten. “ Die Arbeitgeberin kann also je nach Berufserfahrung entscheiden, ob die Probezeit reduziert wird. Die Kriterien bleiben empirisch aber unklar.
} 
dies mit der Vorstellung, wonach gute Arbeit „ein festes, verlässliches Einkommen zu erhalten, unbefristet beschäftigt zu sein“ (Fuchs 2006:8), bedeutet, wird unmittelbar klar, dass dies nicht gegeben ist.

\section{Fortzahlung im Krankheitsfall}

Trotz der ungünstigen Verhältnisse puncto Probezeit, Vertragsdauer und Kettenverträge entscheidet sich Lena P. für die Diplomassistenz an der Universität Fribourg, denn sie möchte unbedingt in ihrem Bereich forschen und eine Doktorarbeit verfassen. Lena muss regelmässig tage- oder zweitageweise zu Hause bleiben, weil sie an einer hartnäckigen Migräne leidet. Während der Zeit der Migräne kann sie nicht arbeiten, da die Kopfschmerzen keinerlei Tätigkeit zulassen. Nach Antritt Ihrer Stelle sieht sie im Personalgesetz des Kantons Fribourg folgenden Passus puncto Lohnfortzahlung im Krankheitsfall von befristet Angestellten:

Die Dauer der Gehaltsfortzahlung beträgt: a) einen Monat, wenn die Mitarbeiterin oder der Mitarbeiter für eine Dauer von einem Jahr oder weniger angestellt ist; $b$ ) drei Monate, wenn die Mitarbeiterin oder der Mitarbeiter für eine Dauer von über einem Jahr angestellt ist und die Arbeitsunfähigkeit im ersten Dienstjahr eintritt; c) sechs Monate, wenn die Arbeitsunfähigkeit im zweiten Dienstjahr eintritt. (Artikel 15, Verordnung über die Lohngarantie des Staatspersonals bei Krankheit und Unfall, VLSKU).

Da Lenas erster Vertrag nur über 12 Monate abgeschlossen wurde, kann sie lediglich einen Monat Gehaltsfortzahlung erwarten. Das bedeutet für sie, dass mit ihren zu erwartenden 24 Tagen Migräneausfall nichts mehr dazwischenkommen darf, sonst würde die Lohnfortzahlung eingestellt werden. Erst wenn der zweite Vertrag abgeschlossen wird, kann sie mit drei Monaten Lohnfortzahlung rechnen. Die Ungleichbehandlung gegenüber jenen, die durch ihre Befristung schon unter prekären Arbeitsverhältnissen arbeiten, wird deutlich, wenn man sich die grosszügige Regelung für Mitarbeiterinnen und Mitarbeiter des Staates Fribourg mit unbefristetem Vertrag vor Augen führt: „In den Genuss der Lohngarantie während 730 Tagen (vollständige Lohngarantie) kommen Mitarbeiterinnen und Mitarbeiter mit einem unbefristeten Arbeitsvertrag oder mit einem befristeten Arbeitsvertrag für mindestens zwei Jahre.“ (Artikel 2, VLSKU) Hier zeigt sich auch eine unglückliche Verquickung des Reglements über die wissenschaftlichen Mitarbeiterinnen und Mitarbeiter der Universität und dem Personalgesetz des Kantons. Da ersteres einen sehr kurzen Vertrag zu Beginn vorsieht (7-15 Monate) und letzteres eine Beschränkung der Lohnfortzahlung für Verträge bis zu einem Jahr vorschreibt, kommt zu der prekären Lage eines kurzen Vertrages mit langer Probezeit noch das Damoklesschwert des Lohnverlustes durch eine etwas längere Erkrankung hinzu.

\section{Erkrankung während der Anstellung in einem Drittmittelprojekt}

Lena kommt ohne grössere Probleme durch das erste Jahr und es bietet sich ihr die Möglichkeit, in einem vom Schweizerischen Nationalfonds (SNF) finanzierten Projekt Ihre Dissertation zu schreiben. Das Thema des Projektes ist so nah an dem ihrer Dissertation, dass sie nicht zwei 
Mal überlegen muss, sie wechselt in das Projekt. Die Laufzeit des Projektes beträgt drei Jahre, Zeit genug also, um die Dissertation schreiben zu können, an der sie schon ein Jahr arbeitet. Das Projekt läuft sehr gut, bis sie nach etwa zweieinhalb Jahren krank wird. Lena hat sich einen Virus eingefangen, der sie für mehrere Monate ausser Gefecht setzt. Sie kann nicht mehr arbeiten, aber da sie einen längerfristigen Vertrag hat, sieht die Verordnung des Kantons vor, dass sie bis zu 730 Tage weiterbezahlt wird. Die Krankheit stellt sich als hartnäckiger heraus, als sie vermutet hatte, und sie kann bis zum offiziellen Ende des Projektes nicht arbeiten. Die Universität hat aus den SNF-Mitteln den Lohn sichergestellt, aber die Zeit des Projektes ist nun abgelaufen und das Projekt und ihre Dissertation sind nicht vollendet. Die Doktorandin wendet sich an die Universität und erkundigt sich, ob die Mittel, die genutzt wurden, um die Lohnfortzahlung zu gewährleisten, von der Universität finanziert werden können, aber die Personalverwaltung gibt ihr den Bescheid, dass für Drittmittel-Angestellte auch im Falle der Krankheit die Mittel aus dem Projekt genutzt werden müssen. Der SNF stellt sich allerdings auf den Standpunkt, dass im Falle einer Krankheit die Institution, an der sie angestellt ist, für die Mittel der Fortzahlung verantwortlich zeigen müsse. Das Projekt könne lediglich kostenneutral verlängert werden; ein Aufschub der Abgabe des Endberichts sei demnach möglich, aber keine Finanzierung der Ausfallzeit, so der SNF.

Ganz offensichtlich liegt die Praxis der Fortzahlung im Krankheitsfall von Drittmittel-Angestellten der Universität über Kreuz mit der Praxis des SNF, was die Unsicherheit der Angestellten sehr stark erhöht. Eine befristete Angestellte, die aus Drittmitteln finanziert wird, dann allerdings längere Zeit krank wird und aus anderen Mitteln als jenen des Drittmittelgebers finanziert werden muss, ist nicht vorgesehen. Dies erscheint aussergewöhnlich, weil Drittmittelprojekte sehr häufig mit Globalbudgets arbeiten, die nicht überschritten werden dürfen. Wie die Doktorandin ihre Qualifizierungsarbeit ohne weitere Mittel fertigstellen soll, bleibt ihr überlassen; das gleiche gilt für die Beendigung des Projektes. Die Unsicherheit, die hier für die Angestellte entsteht, ist mit dem Konzept Gute Arbeit nicht vereinbar.

\section{Fazit}

Es wurde anhand eines Beispiels versucht zu zeigen, dass die Form der mehrfach befristeten Anstellungsverträge, die Regelung der Probezeit, die Organisation der Lohnfortzahlung im Krankheitsfall sowie im Falle einer Anstellung mit Drittmitteln im Verbund zu prekärer Beschäftigung von befristet angestellten Mitarbeiterinnen und Mitarbeitern an der Universität führt. All diese - möglicherweise nicht intendierten Nebeneffekte - widersprechen grundlegend den Konzepten Decent Work und Gute Arbeit. Um dieser Prekarisierung von befristet Beschäftigten an der Universität entgegen zu wirken sind aus meiner Sicht folgende Schritte notwendig:

- Die Erstbefristung eines Vertrages auf 7-15 Monate ist abzuschaffen und durch eine durchgängige Anstellung für die vorgesehenen fünf Jahre zu ersetzen.

- Die exzessive Probezeit im Kanton Fribourg ist dem Obligationenrecht anzupassen. Dies würde bedeuten, es gäbe eine normale Probezeit von einem Monat, in begründeten Fällen von 3 Monaten. 
- Die Fortzahlung im Krankheitsfall muss schon ab dem ersten Jahr auf mindestens 3 Monate angepasst werden. Besser wäre es noch, man würde eine einheitliche Regelung für befristete und festangestellte MitarbeiterInnen anstreben. Es erscheint nicht nachvollziehbar, dass Angestellte mit dem Nachteil der befristeten Beschäftigung auch noch den Nachteil der kürzeren Fristen der Lohnfortzahlung im Krankheitsfall tragen müssen.

- Die Universität muss im Falle einer Erkrankung die Lohnfortzahlung von DrittmittelAngestellten sicherstellen. Es kann nicht sein, dass der ungelöste Konflikt zwischen Universität und Drittmittelgebern auf dem Rücken der Projektangestellten ausgetragen wird.

Befristete Beschäftigung an den Universitäten ist immer mit Unsicherheit verbunden, insbesondere deswegen, weil der Karrierepfad innerhalb der Academia aufgrund der wenigen festen Stellen in diesem Bereich alles andere als vorgespurt ist. Es ist aus meiner Sicht wenig hilfreich, wenn dieser strukturellen Unsicherheit aufgrund von sehr ungünstigen Regulierungen noch zusätzliche Unsicherheit hinzugefügt wird. Der Kanton Fribourg hat den 17 Zielen nachhaltiger Entwicklung der Agenda nachhaltige Entwicklung der Vereinten Nationen 2030 zugestimmt, darin ist auch das Ziel der Decent Work enthalten. Meines Erachtens sollte dieses Ziel auch in den Regulierungen der Arbeitnehmerinnen und Arbeitnehmer des Kantons verwirklicht werden. Das übrigens nicht nur, um den Arbeitnehmerinnen und Arbeitnehmern prekäre Arbeitsverhältnisse zu ersparen, sondern auch um attraktive Arbeitsplätze zu schaffen, auf denen die klügsten Köpfe auch gerne arbeiten möchten.

\section{Danksagung}

Der Autor bedankt sich bei den anonymen GutachterInnen und der Redaktion des Journals sozialpolitik.ch für die wertvollen Hinweise und Kommentare.

\section{Deklaration von Interessenkonflikten}

Der Autor deklariert keine Interessenkonflikte in Bezug auf Forschung, Autorenschaft und/oder Publikation des Artikels.

\section{Finanzierung}

Der Autor hat keine finanzielle Unterstützung für die Forschung, die Autorenschaft und die Publikation dieses Artikels erhalten.

\section{Literaturverzeichnis}

Doktoratsreglement Mathematisch-Naturwissenschaftliche und Medizinische Fakultät der Universität Fribourg. Zugriff am 03.06.20 auf https://www3.unifr.ch/apps/legal/de/document/927509. 
Ferraro, Tânia, Nuno Rebelo dos Santos, Leonor Pais and Lisete Mónico (2016). Historical Landmarks of Decent Work. European Journal of Applied Business and Management, 2(1), 77-96.

Fuchs, Tatjana (2006). Was ist Gute Arbeit? Anforderungen aus Sicht von Erwerbstätigen. INQA-Bericht Nr. 19. Dortmund: INQA.

Gesetz über das Staatspersonal. Zugriff am 03.06.20 auf https://bdlf.fr.ch/app/de/texts of law/1 22.70.1/versions/4755.

International Labour Organization (1944). Declaration concerning the aims and purposes of the International Labour Organisation (Philadelphia Declaration). Philadelphia: International Labour Office.

International Labour Organization (1946). Instrument for the amendment of the Constitution of the International Labour Organisation. Montreal: International Labour Office.

International Labour Organization (1999). Decent Work: Report of the Director-General at $87^{\text {th }}$ Session of International Labour Conference, June. Geneva: International Labour Office.

Obligationenrecht. Bundesgesetz betreffend die Ergänzung des Schweizerischen Zivilgesetzbuches (Fünfter Teil: Obligationenrecht). Zugriff am 07.08.20 auf https://www.admin.ch/opc/de/classifiedcompilation/22.html.

Reglement über die wissenschaftlichen Mitarbeiterinnen und Mitarbeiter der Universität Fribourg. Zugriff am 03.06.20 auf https://www3.unifr.ch/apps/legal/de/document/274860.

Schmucker, Rolf (2020). Von „Decent Work“ zu „Guter Arbeit“. Zur Bedeutung arbeitspolitischer Leitbilder. sozialpolitik.ch 2/2020 - Artikel 2.2.

United Nations (2015a). Universal Declaration of Human Rights. Internet-Site. Zugriff am 03.06.20 auf https://www.un.org/en/udhrbook/pdf/udhr booklet en web.pdf.

United Nations (2015b). Sustainable Development Goals. Internet-Site. Zugriff am 03.06.20 auf https://sustainabledevelopment.un.org/sdgs.

Verordnung über die Lohngarantie des Staatspersonals bei Krankheit und Unfall. Zugriff am 03.06.20 auf https://bdlf.fr.ch/app/de/texts of law/122.72.18. 